\title{
Pseudo-Acanthosis nigricans bei einem 12 jahre aliten Jungen nach Nierentransplantation
}

\section{Ch. v. Schnakenburg1', B. Enke1, K. Jürgens², G. Offner}

1 Zentrum für Kinderheilkunde, Abt. für Nieren- und Stoffwechselkrankheiten, Medizinische Hochschule Hannover

2 Hautklinik, Universitätskrankenhaus Eppendorf, Hamburc

\section{Zusammenfassung}

Hautveränderungen unter immunsuppressiver Therapie bedürfen einer spezifischen Diaqnostik. Wir stellen den Fall eines 12 lahre alter |ungen vor, der sich 3 Monate nach Nierentransplantation mit einer Pseudo-Acanthosis nigricans vorstellte.

Pseudo-Acanthosis nigricans in a 12 year old boy after kidney transplantation

Cutaneous lesions under immunosuppressive therapy require a specific diagnostic approach. We present the case of a 12 year old boy who developed pseudo-acanthosis nicricans 3 months after renal transplantation.

Anamnese

Ein 12-jähriger Junge stellte sich 3 Monate nach erfolgreicher Nierentransplantation im Rahmen einer Routinekontrolle mit bräunlich-schmutzigen Hautveränderungen an mechanisch stärker exponierten Arealen vor, die zum Teil durch Waschen abgebürstet worden waren. Als Grunderkrankung der vorherigen Niereninsuffizienz lag eine obstruktive Uropathie vor, als deren Ursache Urethralklappen im Säuglingsalter problemlos endoskopisch entfernt worden waren.

\section{Untersuchungsbefund}

12 Jahre alter Junge in gutem Allgemein- und Ernährungszustand, Gewicht 41 kg (P 25), Länge 156 cm (P 25). Internistischer Untersuchungsbefund unauffällig bis auf leichte Hypertrichose und reizlose Narbe nach Nierentransplantation im rechten Unterbauch. Bräunliche, unscharf becrenzte, leicht erhabene Hautveränderungen im Bereich der Armbeugen, des Halses sowie periumbilikal (Abb. 1). Kein Juckreiz und keine Kratzspuren. Keine Lymphadenopathie.

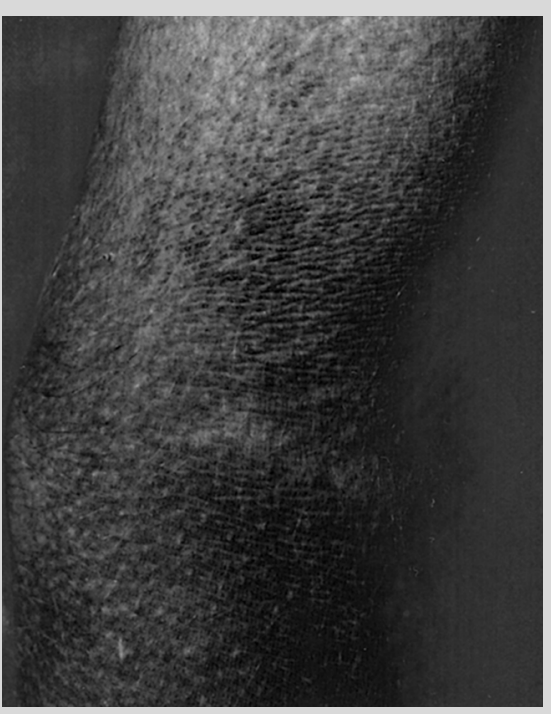

Abb. 1 Hautbe-

Nierentransplanta-

tion: schmutzic

bräunliche Hyper piamentierung in der Ellenbeuge.

\section{Labor}

Unauffälliges Differenzialblutbild, negative Infektparameter, eingeschränkte GFR mit $40 \mathrm{ml} / \mathrm{min} / 1,73 \mathrm{~m}^{2}$ (S-Kreatinin 1,6 mg/dl). Normalwerte für Nüchternblutzucker, HbA 1 und C-Peptid, leicht erhöhtes Nüchtern-Insulin ( $32 \mathrm{mU} / \mathrm{l}$, bei Kontrolle normal), unauffälliger oraler Glukose-Toleranztest mit adäquatem Insulinanstiec auf $180 \mathrm{mU} / \mathrm{I}$ nach 30 Minuten. Ciclosporin-Talspiegel um $150 \mathrm{ng}$ $\mathrm{ml}$ bei einer Dosis von $150 \mathrm{mg} / \mathrm{m}^{2}$ in 2 Einzelgaben. Die weitere Medikation bestand aus Prednisolon $\left(4 \mathrm{mg} / \mathrm{m}^{2}\right)$ sowie Metoprolol, Dihydralazin und Clonidin zur Einstellung eines arteriellen Hypertonus

Therapie und Verlauf

Im Rahmen einer Nephrektomie der Eigennieren konnte ir Narkose eine Biopsie der Hautläsionen aus der Nähe der Transplantatnarbe entnommen werden, mit der die Verdachtsdiagnose einer Pseudo-Acanthosis niqricans bestätiqt werden konnte (Abb. 2).

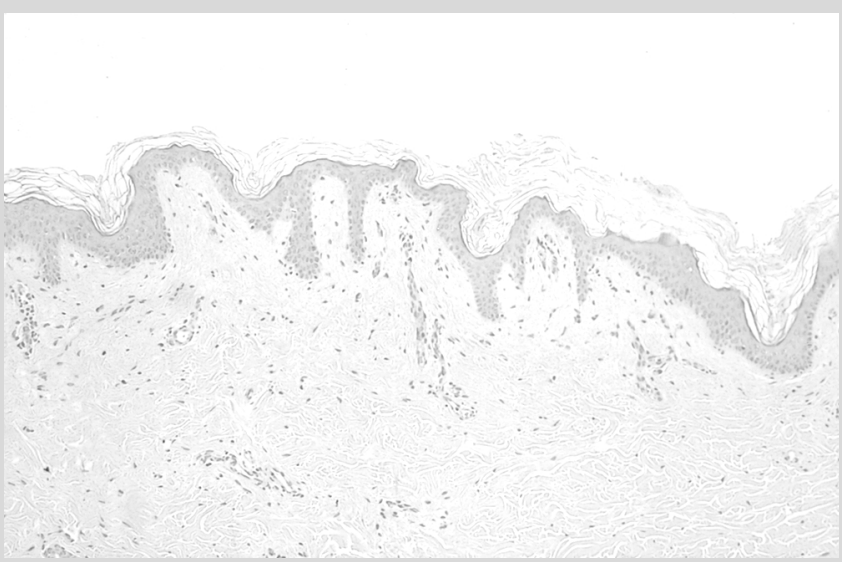

Abb.2 Histologischer Befund: Epidermale Papillomatose mit milde Acanthose sowie Orthohyperkeratose. 


\section{VISITE Klin Pädiatr 2001; 213: 288}

\section{Zusammenfassung}

Pseudo-Acanthosis nigricans in a 12 year old boy after kidney transplantation

Anamnese

Labor

Untersuchungsbefund

Therapie und Verlauf
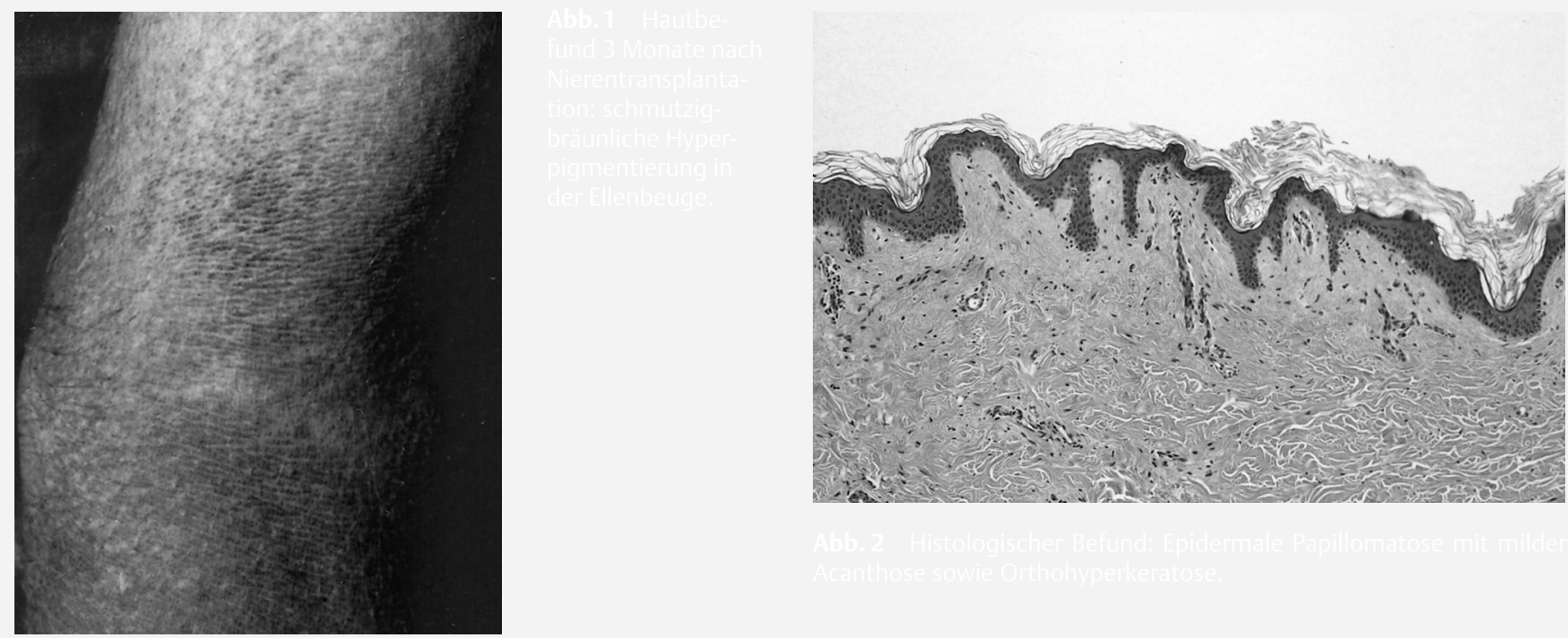

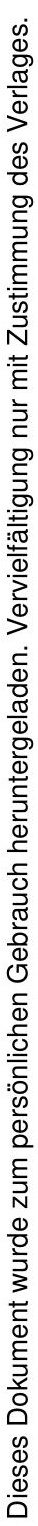




\section{VISITE Klin Pädiatr 2001; 213: 288}

\section{Zusammenfassung}

Pseudo-Acanthosis nigricans in a 12 year old boy after kidney transplantation

Anamnese

Labor

Untersuchungsbefund

Therapie und Verlauf
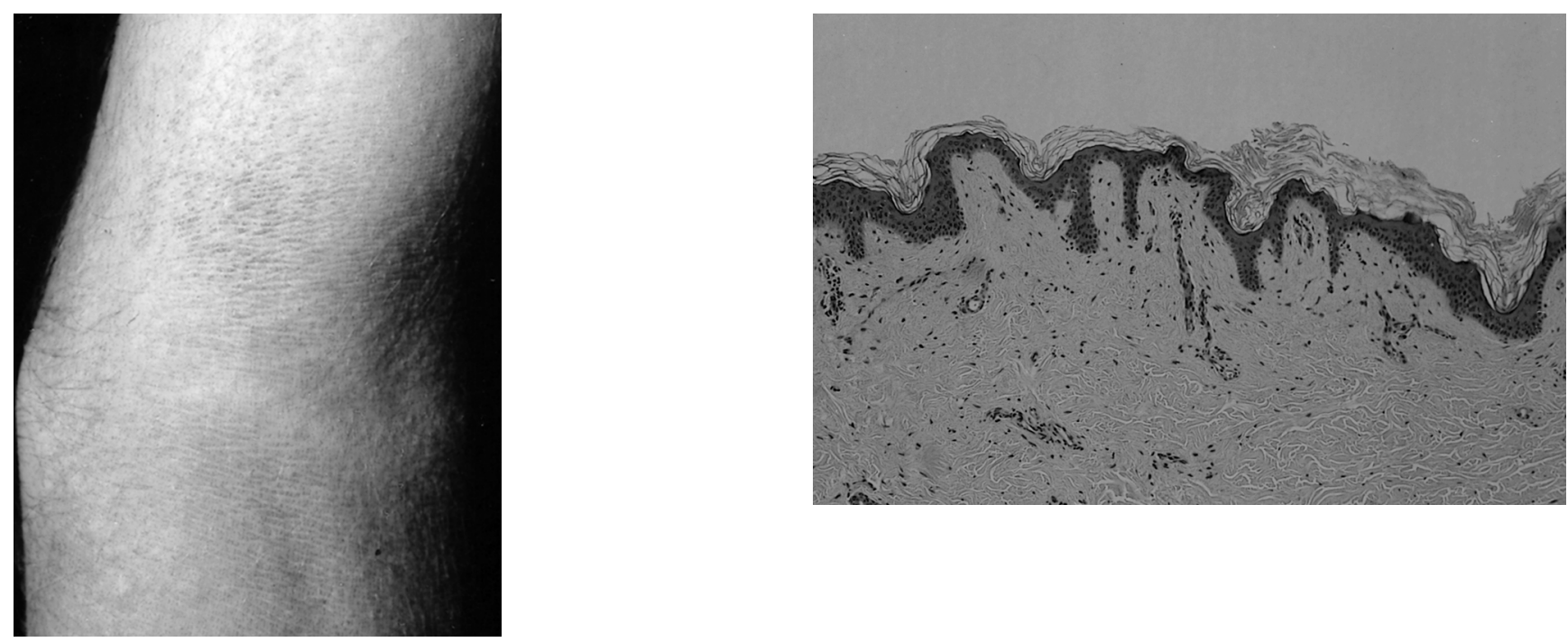


\title{
Pseudo-Acanthosis nigricans bei einem 12 Jahre alten Jungen nach Nierentransplantation
}

\author{
Ch. v. Schnakenburg ${ }^{1}$, B. Enke¹, K. jürgens², G. Offner \\ 1 Zentrum für Kinderheilkunde, Abt. für Nieren- und Stoffwechselkrankheiten, Medizinische Hochschule Hannover \\ 2 Hautklinik, Universitätskrankenhaus Eppendorf, Hamburg
}

\section{Zusammenfassung}

Hautveränderungen unter immunsuppressiver Therapie bedürfen einer spezifischen Diagnostik. Wir stellen den Fall eines 12 Jahre alten Jungen vor, der sich 3 Monate nach Nierentransplantation mit einer Pseudo-Acanthosis nigricans vorstellte.

\section{Pseudo-Acanthosis nigricans in a 12 year old boy after kidney transplantation}

Cutaneous lesions under immunosuppressive therapy require a specific diagnostic approach. We present the case of a 12 year old boy who developed pseudo-acanthosis nigricans 3 months after renal transplantation.

\section{Anamnese}

Ein 12-jähriger Junge stellte sich 3 Monate nach erfolgreicher Nierentransplantation im Rahmen einer Routinekontrolle mit bräunlich-schmutzigen Hautveränderungen an mechanisch stärker exponierten Arealen vor, die zum Teil durch Waschen abgebürstet worden waren. Als Grunderkrankung der vorherigen Niereninsuffizienz lag eine obstruktive Uropathie vor, als deren Ursache Urethralklappen im Säuglingsalter problemlos endoskopisch entfernt worden waren.

\section{Untersuchungsbefund}

12 Jahre alter Junge in gutem Allgemein- und Ernährungszustand, Gewicht 41 kg (P 25), Länge 156 cm (P 25). Internistischer Untersuchungsbefund unauffällig bis auf leichte Hypertrichose und reizlose Narbe nach Nierentransplantation im rechten Unterbauch. Bräunliche, unscharf begrenzte, leicht erhabene Hautveränderungen im Bereich der Armbeugen, des Halses sowie periumbilikal (Abb.1). Kein Juckreiz und keine Kratzspuren. Keine Lymphadenopathie.

\section{abor}

Unauffälliges Differenzialblutbild, negative Infektparameter, eingeschränkte GFR mit $40 \mathrm{ml} / \mathrm{min} / 1,73 \mathrm{~m}^{2}$ (S-Kreatinin 1,6 mg/dl). Normalwerte für Nüchternblutzucker, $\mathrm{HbA}_{1 \mathrm{C}}$ und C-Peptid, leicht erhöhtes Nüchtern-Insulin (32 mU/l, bei Kontrolle normal), unauffälliger oraler Glukose-Toleranztest mit adäquatem Insulinanstieg auf $180 \mathrm{mU} / \mathrm{I}$ nach 30 Minuten. Ciclosporin-Talspiegel um $150 \mathrm{ng} /$ $\mathrm{ml}$ bei einer Dosis von $150 \mathrm{mg} / \mathrm{m}^{2}$ in 2 Einzelgaben. Die weitere Medikation bestand aus Prednisolon $\left(4 \mathrm{mg} / \mathrm{m}^{2}\right)$ sowie Metoprolol, Dihydralazin und Clonidin zur Einstellung eines arteriellen Hypertonus.

\section{Therapie und Verlauf}

Im Rahmen einer Nephrektomie der Eigennieren konnte in Narkose eine Biopsie der Hautläsionen aus der Nähe der Transplantatnarbe entnommen werden, mit der die Verdachtsdiagnose einer Pseudo-Acanthosis nigricans bestätigt werden konnte (Abb. 2).
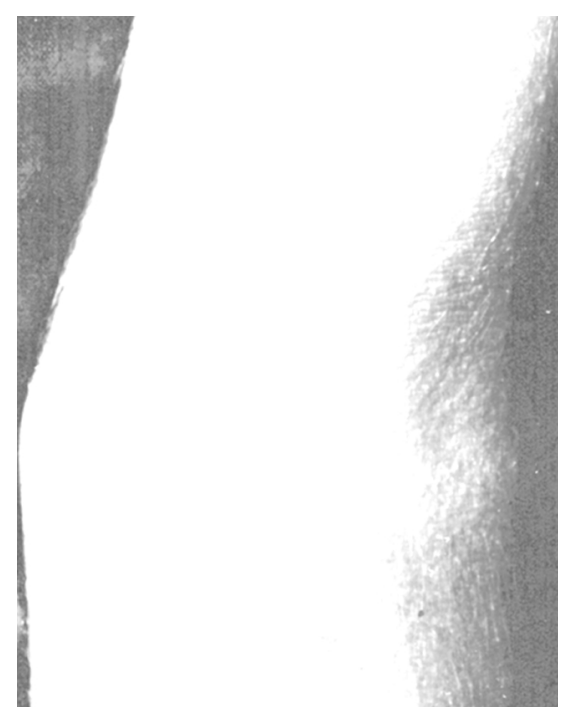

Abb. 1 Hautbefund 3 Monate nach Nierentransplantation: schmutzigbräunliche Hyperpigmentierung in der Ellenbeuge.
Abb. 2 Histologischer Befund: Epidermale Papillomatose mit milder Acanthose sowie Orthohyperkeratose. 
Unter einer keratolytischen Therapie mit 10\% Natrium-Chlorat verschwanden die Hautveränderungen innerhalb von wenigen Tagen. Allerdings kam es in den folgenden 2 Jahren, besonders im Winter bei geringerer Sonnenexposition, zu milden Rezidiven, die jeweils rasch auf dieselbe Therapie ansprachen. Hinweise für eine prädiabetische Stoffwechsellage oder für lymphoproliferative Erkrankungen ergaben sich auch im Langzeitverlauf nicht.

\section{Diagnose}

Pseudo-Acanthosis nigricans unter immunsuppressiver Therapie.

\section{Kommenta}

Klinisch werden verschiedene Formen der Acanthosis nigricans unterschieden: eine benigne oder juvenile Form mit unregelmäßicer dominanter Vererbung; eine maliqne, paraneoplastische Form, die häufig mit Adenokarzinomen bei Erwachsenen assoziiert ist; syndromatische Formen wie bei Hirschowitz- oder Rabson-Medenhall-Syndrom und polyzystischen Ovarien; mit Übergewicht assoziierte sowie Medikamenten-induzierte Formen nach Einnahme von Nikotinamid, Stilboestrol, Folsäureantaconisten und Immunsuppressiva [1]. Im deutschsprachigen Schrifttum werden die letztgenannten erworbenen Formen als PseudoAcanthosis nigricans bezeichnet [2]. Die Prävalenz der Acanthosis nigricans unterscheidet sich wesentlich in verschiedenen ethnischen Gruppen. Sie ist am niedrigsten bei Mitteleuropäern (0,5\%) und am höchsten bei einigen nordamerikanischen Indianerstämmen $(30-40 \%)$ [3]. Acanthosis nigricans ist als wesentlicher Marker für Hyperinsulinismus und Typ-\|l-Diabetes mellitus beschrieben worden [4]

Histologische Untersuchungen zeigen wie im vorgestellten Fall eine Hyperkeratose, epidermale Papillomatose und milde Akanthose [5]. Nahezu in allen Fällen sind die betroffenen Hautstellen gegenüber vermehrter mechanischer Belastung durch Kleidung oder Hautfalten exponiert. Als Ursache für die Hautveränderungen wird eine verminderte Viskosität der Extrazellulärmatrix durch veränderte Glykosaminoglykane diskutiert [6]

Die immunsuppressive Therapie mit Steroiden und Ciclosporin A ist ein bekannter Risikofaktor, einen Diabetes mellitus zu entwickeln $[7,8]$. Hinweise dafür ergaben sich bei dem vorgestellten Patienten jedoch auch bei wiederholten Untersuchungen im Langzeitverlauf nicht. Wir spekulieren, dass die Acanthosis nigricans durch die initial nach Nierentransplantation höheren Steroidgaben (Prednisolon $60 \mathrm{mg} / \mathrm{m}^{2}$ ) ausgelöst und durch die Therapie mit Ciclosporin A unterhalten werden. Insgesamt haben wir in den vergangenen 2 Jahren noch bei zwei weiteren nierentransplantierten Jugendlichen identische Verläufe über einen Zeitraum von mehreren Monaten beobachtet, ohne dass sich Hinweise für eine gestörte Glukosetoleranz oder Malignität nachweisen ließen.

\section{Literatur}

Schwartz RA, Janniger CK. Childhood acanthosis nigricans. Cutis $1995 ; 55: 337-341$

Schmoeckel C. Lexikon und Differentialdiagnose der Dermatologie 5. Aufl. Thieme, Stuttgart 1994; 88-99

Stuart CA. Gilkinson CR, Smith MM, Bosma AM, Keenan BS, Nagamani M. Acanthosis nigricans as a risk factor for non-insulin dependent diabetes mellitus. Clin Pediatr 1998; 37: 73 - 79

${ }^{4}$ Brockow K, Steinkraus V, Rinninger F, Abeck D, Ring J. Acanthosis nigricans: a marker for hyperinsulinemia. Pediatr Dermatol 1995; 12 $323-326$

Lang E, Schmidt R. Beziehungen zwischen Acanthosis nigricans benigna und Pseudoacanthosis nigricans. Hautarzt 1982; 33: 640 645

Stone Ol. Acanthosis nicricans - decreased extracellular matrix viscosity: cancer, obesity, diabetes, corticosteroids, somatropin. Med Hypotheses 1993; 49: $154-157$

Fennell RS, Van Deusen J, Riley W. Steroid-induced diabetes in pediatric renal transplant recipients. Int | Pediatr Nephrol 1983; 4 : $103-107$

8 Yamamoto H. Akazawa S. Yamaquchi Y. Yokota A. Yamasaki H. Nakanishi T, Tahara D, Matsuya F, Saito Y, Nagataki S. Effects of cyclosporin A and low dosages of steroid on posttransplantation diabetes in kidney transplant recipients. Diabetes Care 1991; 14 $867-870$

Dr. med. Christian v. Schnakenburg

Zentrum für Kinderheilkunde

Medizinische Hochschule Hannover

30623 Hannove

E-mail: c.schnakenburg@web.de 


\section{Literatur}

Diagnose

Kommentar 

Unter einer keratolytischen Therapie mit 10\% Natrium-Chlorat verschwanden die Hautveränderungen innerhalb von wenigen Tagen. Allerdings kam es in den folgenden 2 Jahren, besonders im Winter bei geringerer Sonnenexposition, zu milden Rezidiven, die jeweils rasch auf dieselbe Therapie ansprachen. Hinweise für eine prädiabetische Stoffwechsellage oder für lymphoproliferative Erkrankungen ergaben sich auch im Langzeitverlauf nicht.

\section{Diagnose}

Pseudo-Acanthosis nigricans unter immunsuppressiver Therapie.

\section{Kommentar}

Klinisch werden verschiedene Formen der Acanthosis nigricans unterschieden: eine benigne oder juvenile Form mit unregelmäßiger dominanter Vererbung; eine maligne, paraneoplastische Form, die häufig mit Adenokarzinomen bei Erwachsenen assoziiert ist; syndromatische Formen wie bei Hirschowitz- oder Rabson-Medenhall-Syndrom und polyzystischen Ovarien; mit Übergewicht assoziierte sowie Medikamenten-induzierte Formen nach Einnahme von Nikotinamid, Stilboestrol, Folsäureantagonisten und Immunsuppressiva [1]. Im deutschsprachigen Schrifttum werden die letztgenannten erworbenen Formen als PseudoAcanthosis nigricans bezeichnet [2]. Die Prävalenz der Acanthosis nigricans unterscheidet sich wesentlich in verschiedenen ethnischen Gruppen. Sie ist am niedrigsten bei Mitteleuropäern (0,5\%) und am höchsten bei einigen nordamerikanischen Indianerstämmen (30-40\%) [3]. Acanthosis nigricans ist als wesentlicher Marker für Hyperinsulinismus und Typ-II-Diabetes mellitus beschrieben worden [4].

Histologische Untersuchungen zeigen wie im vorgestellten Fall eine Hyperkeratose, epidermale Papillomatose und milde Akanthose [5]. Nahezu in allen Fällen sind die betroffenen Hautstellen gegenüber vermehrter mechanischer Belastung durch Kleidung oder Hautfalten exponiert. Als Ursache für die Hautveränderungen wird eine verminderte Viskosität der Extrazellulärmatrix durch veränderte Glykosaminoglykane diskutiert [6].

Die immunsuppressive Therapie mit Steroiden und Ciclosporin A ist ein bekannter Risikofaktor, einen Diabetes mellitus zu entwickeln $[7,8]$. Hinweise dafür ergaben sich bei dem vorgestellten Patienten jedoch auch bei wiederholten Untersuchungen im Langzeitverlauf nicht. Wir spekulieren, dass die Acanthosis nigricans durch die initial nach Nierentransplantation höheren Steroidgaben (Prednisolon $60 \mathrm{mg} / \mathrm{m}^{2}$ ) ausgelöst und durch die Therapie mit Ciclosporin A unterhalten werden. Insgesamt haben wir in den vergangenen 2 Jahren noch bei zwei weiteren nierentransplantierten Jugendlichen identische Verläufe über einen Zeitraum von mehreren Monaten beobachtet, ohne dass sich Hinweise für eine gestörte Glukosetoleranz oder Malignität nachweisen ließen.

\section{Literatur}

${ }^{1}$ Schwartz RA, Janniger CK. Childhood acanthosis nigricans. Cutis 1995; 55: 337-341

2 Schmoeckel C. Lexikon und Differentialdiagnose der Dermatologie, 5. Aufl. Thieme, Stuttgart 1994; 88-99

${ }^{3}$ Stuart CA, Gilkinson CR, Smith MM, Bosma AM, Keenan BS, Nagamani M. Acanthosis nigricans as a risk factor for non-insulin dependent diabetes mellitus. Clin Pediatr 1998; 37: 73 - 79

${ }^{4}$ Brockow K, Steinkraus V, Rinninger F, Abeck D, Ring J. Acanthosis nigricans: a marker for hyperinsulinemia. Pediatr Dermatol 1995; 12: $323-326$

${ }^{5}$ Lang E, Schmidt R. Beziehungen zwischen Acanthosis nigricans benigna und Pseudoacanthosis nigricans. Hautarzt 1982; 33: $640-$ 645

${ }^{6}$ Stone OJ. Acanthosis nigricans - decreased extracellular matrix viscosity: cancer, obesity, diabetes, corticosteroids, somatropin. Med Hypotheses 1993; 49: 154-157

${ }^{7}$ Fennell RS, Van Deusen J, Riley WJ. Steroid-induced diabetes in pediatric renal transplant recipients. Int J Pediatr Nephrol 1983; 4: $103-107$

${ }^{8}$ Yamamoto $\mathrm{H}$, Akazawa S, Yamaguchi Y, Yokota A, Yamasaki $\mathrm{H}$, Nakanishi T, Tahara D, Matsuya F, Saito Y, Nagataki S. Effects of cyclosporin A and low dosages of steroid on posttransplantation diabetes in kidney transplant recipients. Diabetes Care 1991; 14 $867-870$

Dr. med. Christian v. Schnakenburg

Zentrum für Kinderheilkunde

Medizinische Hochschule Hannover 30623 Hannover

E-mail: c.schnakenburg@web.de 